Waves in Random and Complex Media

February 2008, Volume 18, Issue 1, pages 185 - 196

http://dx.doi.org/10.1080/17455030701564644

(C) 2008 Taylor \& Francis Ltd.
Archimer http://www.ifremer.fr/docelec/ Archive Institutionnelle de l'Ifremer

The original publication is available at http://www.tandf.co.uk/journals/

\title{
Predicted Doppler shifts induced by ocean surface wave displacements using asymptotic electromagnetic wave scattering theories
}

\author{
A. A. Mouche ${ }^{\mathrm{a}, \mathrm{c}} ;$ B. Chapron ${ }^{\mathrm{c}} ;$ N. Reul ${ }^{\mathrm{c}, *} ;{ }^{*}$ F. Collard ${ }^{\mathrm{b}}$ \\ ${ }^{a}$ Centre National d'Etudes Spatiales, Paris, France \\ bOOST Technologies, Plouzané, France \\ cIfremer, Pointe du Diable, Plouzané, 29280, France \\ *: Corresponding author : N. Reul, email address : Nicolas.Reul@ifremer.fr
}

\begin{abstract}
:
Sea surface motions can produce different measured Doppler shifts with respect to instrumental configurations (incidence angle, electromagnetic wavelength, polarization). Under Gaussian statistics for the sea surface elevation and in the general framework of asymptotic theories for ocean surface electromagnetic wave scattering, Doppler shifts can be predicted. The small-slope, Kirchhoff, local curvature and resonant curvature approximations are compared in the backscatter configuration. Predicted Doppler shifts for Kirchhoff and small-slope approximations in co-polarized configuration are insensitive to the polarization state. On the other hand, the local and resonant curvature solutions, through a phase perturbation formalism, yield to significant differences between co-polarized predicted Doppler shifts. Comparisons with data are shown to confirm the polarization and wind speed sensitivities.
\end{abstract}

\section{1 introduction}

It has been recently shown [1,2] that Doppler frequency anomalies, i.e. the difference between the measured and the geometrically predicted Doppler shifts, can be systematically extracted from satellite-borne Synthetic Aperture Radar (SAR) measurements over ocean scenes. As analyzed, these Doppler shifts are directly associated to the distributed motions of the dißerent ocean surface roughness scales participating to the scattered signals. As already reported, under the actions of wind and current, sea surface roughness elements, in a 
very short time, can evolve sufficiently rapidly to help mapping instantaneous motions toward the receiving antenna.

There are very practical interests to obtain direct surface velocity measurements from space, and potential and limitations of using SAR measurements must be discussed. As foreseen, the use of direct measurements can certainly help the development of consistent inversions of instantaneous sea surface geometry and kinematic properties. However, questions regarding the sensor physics and optimal instrumental configurations (incidence angle, wavelength, polarization) must be answered to help the analysis of present measurements and the design of future satellite missions.

To advance in such investigations, our purpose in this paper is to use and apply the latest improvements suggested in the field of the asymptotic electromagnetic modeling. Predictions will help to better understand the wavelength, incidence angle, and polarization sensitivities of time dependent ocean surface radar backscattered signals. Under Gaussian statistics, time dependent asymptotic solutions are first derived to define a Doppler frequency and the associated measured motion, section 3. The proposed developments offer simplified tractable numerical solutions discussed and compared with reported measurements, section 4 . Section 5 summarizes and present investigations.

\section{Coordinates system and definitions}

To expose the general problem in this paper, we adopt the same vectorial conventions than in $[3,4]$. The right cartesian coordinate system is defined by the triplet of normalized vectors $(\hat{x}, \hat{y}, \hat{z})$, where the z-axis is directed upward. $\Sigma$ is the rough surface which separates the upper medium and the lower medium (respectively air and water in our specific case). The (sea) surface elevation is represented by $z=\eta(x, y, t)=\eta(\boldsymbol{r}, t)$, where $\boldsymbol{r}$ is the horizontal component of the three-dimensional position wave vector $\boldsymbol{R}=(\boldsymbol{r}, z)$ and $t$ the time dependence of the sea surface. The sea surface is now defined as:

$$
\eta(\boldsymbol{r}, t)=\int_{\boldsymbol{\xi}} \hat{\eta}(\boldsymbol{\xi}) e^{i(\boldsymbol{\xi} \cdot \boldsymbol{r}-\omega(\boldsymbol{\xi}) t)} d \boldsymbol{\xi},
$$

where $\hat{\eta}(\boldsymbol{\xi})$ is the Fourier transform of the surface elevation, $\boldsymbol{\xi}$ is the wavenumber of the sea surface wave whose wavelength is $\lambda=2 \pi /|\boldsymbol{\xi}|$ and $\omega(\boldsymbol{\xi})$ the pulsation frequency defined according to the dispersion relationship. We consider a incident downward propagating electromagnetic plane wave with a wavevector $\boldsymbol{K}_{0}=\left(\boldsymbol{k}_{0},-q_{0}\right)$. The up-going scattered waves is characterized by the wave-vector $\boldsymbol{K}=\left(\boldsymbol{k}, q_{k}\right) . \boldsymbol{k}_{0}$ and $\boldsymbol{k}$ are the horizontal components of the incident and scattered waves whereas $q_{0}$ and $q_{k}$ are the vertical ones. We define 
also $\boldsymbol{Q}_{h}$ and $Q_{z}$ related to the coordinates of the wave numbers $\boldsymbol{K}$ and $\boldsymbol{K}_{0}$ : $\boldsymbol{Q}_{h}=\boldsymbol{k}-\boldsymbol{k}_{0}$ and $Q_{z}=q_{0}+q_{k}$.

The scattered field above and far away $(R \rightarrow \infty)$ from the sea surface is assumed to be related to the incident wave through the relation:

$$
\boldsymbol{E}_{s}(\boldsymbol{R}, t)=-2 i \pi \frac{e^{i K R}}{R} \mathbb{S}\left(\boldsymbol{k}, \boldsymbol{k}_{0}\right) \cdot \hat{E}_{0}
$$

$\mathbb{S}\left(\boldsymbol{k}, \boldsymbol{k}_{0}\right)$ is the so-called scattering operator. $\boldsymbol{E}_{s}(\boldsymbol{R})$ and $\mathbb{S}\left(\boldsymbol{k}, \boldsymbol{k}_{0}\right)$ can be decomposed on the fundamental polarization basis:

$$
\boldsymbol{p}_{v}^{ \pm}( \pm k)=\frac{k \hat{z} \mp q_{k} \hat{k}}{K} \quad \boldsymbol{p}_{h}^{ \pm}( \pm k)=\hat{z} \times \hat{k}
$$

where the subscripts $v$ and $h$ indicate the vertical and horizontal polarizations, respectively. The minus superscript corresponds to the down-going plane waves while the plus superscript to the up-going waves. In this vectors basis, the scattering operator is related to the scattering amplitude $2 \times 2$ matrix through:

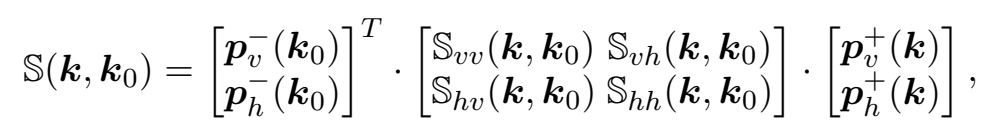

where the superscript $T$ stands for the transpose operator. In the $2 \times 2$ matrix, the first subscript indicates the incident polarization whereas the second one indicates the scattered polarization configuration considered.

For a given polarization configuration $p q, \mathbb{S}^{p q}\left(\boldsymbol{k}, \boldsymbol{k}_{0}\right)$ is written as:

$$
\mathbb{S}^{p q}\left(\boldsymbol{k}, \boldsymbol{k}_{0}\right)=\frac{1}{Q_{z}} \int_{\boldsymbol{r}} \mathbb{N}^{p q}\left(\boldsymbol{k}, \boldsymbol{k}_{0} ; \eta(\boldsymbol{r}, t)\right) e^{-i Q_{z} \eta(\boldsymbol{r}, t)} e^{-i \boldsymbol{Q}_{H} \cdot \boldsymbol{r}} d \boldsymbol{r}
$$

where $\mathbb{N}\left(\boldsymbol{k}, \boldsymbol{k}_{0} ; \eta(\boldsymbol{r}, t)\right)$ is a kernel depending on the approach considered to establish the solution. As already discussed, the kernel expansion can be defined to satisfy the two asymptotic limits given by the small perturbation and the Kirchhoff solutions. A general development of the kernel $\mathbb{N}\left(\boldsymbol{k}, \boldsymbol{k}_{0} ; \eta(\boldsymbol{r}, t)\right)$ writes:

$$
\mathbb{N}\left(\boldsymbol{k}, \boldsymbol{k}_{0} ; \eta(\boldsymbol{r}, t)\right)=\mathbb{N}_{0}\left(\boldsymbol{k}, \boldsymbol{k}_{0}\right)+\mathbb{N}_{1}\left(\boldsymbol{k}, \boldsymbol{k}_{0} ; \eta(\boldsymbol{r}, t)\right)+\mathbb{N}_{2}\left(\boldsymbol{k}, \boldsymbol{k}_{0} ; \eta(\boldsymbol{r}, t)\right)+\ldots(6)
$$


where the sub-kernels up to the second order are expressed as:

$$
\begin{aligned}
& \mathbb{N}_{1}\left(\boldsymbol{k}, \boldsymbol{k}_{0} ; \eta(\boldsymbol{r}, t)\right)=\int_{\boldsymbol{\xi}} N_{1}\left(\boldsymbol{k}, \boldsymbol{k}_{0} ; \boldsymbol{\xi}\right) \hat{\eta}(\boldsymbol{\xi}) e^{i(\boldsymbol{\xi} \cdot \boldsymbol{r}-\omega t)} d \boldsymbol{\xi} \\
& \mathbb{N}_{2}\left(\boldsymbol{k}, \boldsymbol{k}_{0} ; \eta(\boldsymbol{r}, t)\right)=\int_{\boldsymbol{\xi}} \int_{\boldsymbol{\xi}^{\prime}} N_{2}\left(\boldsymbol{k}, \boldsymbol{k}_{0} ; \boldsymbol{\xi}, \boldsymbol{\xi}^{\prime}\right) \hat{\eta}(\boldsymbol{\xi}) \hat{\eta}\left(\boldsymbol{\xi}^{\prime}\right) e^{i\left[\left(\boldsymbol{\xi}^{\prime}+\boldsymbol{\xi}\right) \cdot \boldsymbol{r}-\left(\omega+\omega^{\prime}\right) t\right]} d \boldsymbol{\xi} d \boldsymbol{\xi}^{\prime}(8)
\end{aligned}
$$

and are polarization dependent. As discussed by Irisov [5] for SSA developments, but also valid for LCA [6] or RCA [3] developments, solutions to satisfy the two asymptotic limits can be easily found. In this study, we only consider a development up to the first order, i.e. $\mathbb{N}_{2}\left(\boldsymbol{k}, \boldsymbol{k}_{0} ; \eta(\boldsymbol{r}, t)\right)=0$.

\section{Doppler Shift}

As obtained for sea surface backscatter simulations, the zeroth order contribution of the scattering matrix expansion apparently significantly dominates the solution. Higher order contributions for the scattering matrix expansion can then be introduced using a phase perturbation technique [7]. This has the advantage to simplify the analytical derivation of the second order statistical moment of the scattering matrix and to offer tractable numerical computation $[3,8]$. Under this assumption, the general expansion of the scattering matrix up to first order can be re-written as:

$$
\mathbb{S}\left(\boldsymbol{k}, \boldsymbol{k}_{0}\right)=\mathbb{N}_{0}\left(\boldsymbol{k}, \boldsymbol{k}_{0}\right) \int_{\boldsymbol{r}} e^{-i Q_{z} \Phi\left(\boldsymbol{k}, \boldsymbol{k}_{0} ; \eta(\boldsymbol{r}, t)\right)} e^{-i \boldsymbol{Q}_{H} \cdot \boldsymbol{r}} d \boldsymbol{r},
$$

with:

$$
\Phi\left(\boldsymbol{k}, \boldsymbol{k}_{0} ; \eta(\boldsymbol{r}, t)\right)=\int_{\boldsymbol{\xi}}\left[1+\frac{N_{1}\left(\boldsymbol{k}, \boldsymbol{k}_{0} ; \boldsymbol{\xi}\right)}{\mathbb{N}_{0}\left(\boldsymbol{k}, \boldsymbol{k}_{0}\right)}\right] \hat{\eta}(\boldsymbol{\xi}) e^{i(\boldsymbol{\xi} \cdot \boldsymbol{r}-\omega t)} d \boldsymbol{\xi}
$$

In the following, $\Phi\left(\boldsymbol{k}, \boldsymbol{k}_{0} ; \eta(\boldsymbol{r}, t)\right)$ is considered as a modified sea surface height function and noted $\tilde{\eta}(\boldsymbol{r}, t) . \eta(\boldsymbol{r}, t)$ is assumed stationary, ergodic and to be a zero mean gaussian variable, i.e. $\left\langle e^{\eta}\right\rangle=e^{\left.<\eta^{2}\right\rangle / 2}$. To this approximation order, $\tilde{\eta}(\boldsymbol{r}, t)$ is simply a linearly filtered version of the true surface elevation. Consequently, $\tilde{\eta}(\boldsymbol{r}, t)$ is also a stationary zero-mean Gaussian variable, and the correlation function is defined such as $\langle\tilde{\eta}(\boldsymbol{r}, t) \tilde{\eta}(\boldsymbol{r}+\Delta \boldsymbol{r}, \Delta t)>=\tilde{\rho}(\Delta \boldsymbol{r}, \Delta t)$. To simplify our notation, in the correlation function expression, $\Delta \boldsymbol{r}$ and $\Delta t$ are replaced by $\boldsymbol{r}$ and $t$ respectively. Thus, the normalized radar cross-section 
becomes a function of time and writes:

$$
\sigma_{0}(\theta, \phi, t)=\left|\frac{\mathbb{N}_{0}\left(\boldsymbol{k}, \boldsymbol{k}_{0}\right)}{Q_{z}}\right|^{2} e^{-Q_{z}^{2} \tilde{\rho}(0,0)} \int_{\boldsymbol{r}}\left[e^{-Q_{z}^{2} \tilde{\rho}(\boldsymbol{r}, t)}-1\right] e^{-i \boldsymbol{Q}_{H} \cdot \boldsymbol{r}} d \boldsymbol{r},
$$

with:

$$
\tilde{\rho}(\boldsymbol{r}, t)=\int_{\boldsymbol{\xi}}\left|1+\frac{N_{1}\left(\boldsymbol{k}, \boldsymbol{k}_{0} ; \boldsymbol{\xi}\right)}{\mathbb{N}_{0}\left(\boldsymbol{k}, \boldsymbol{k}_{0}\right)}\right|^{2} S(\boldsymbol{\xi}) e^{i(\boldsymbol{\xi} \cdot \boldsymbol{r}-\omega t)} d \boldsymbol{\xi}=\int_{\boldsymbol{\xi}} \tilde{S}(\boldsymbol{\xi}) e^{i(\boldsymbol{\xi} \cdot \boldsymbol{r}-\omega t)} d \boldsymbol{\xi},
$$

where $N_{1}\left(\boldsymbol{k}, \boldsymbol{k}_{0}, \boldsymbol{\xi}\right)$ and $\mathbb{N}_{0}\left(\boldsymbol{k}, \boldsymbol{k}_{0}\right)$ depend upon the asymptotic model considered. $\tilde{\rho}(\boldsymbol{r}, t)$ is the correlation function of a filtered surface height spectrum $\tilde{S}(\boldsymbol{\xi})$.

To express the correlation function as a function of the time from a given state, we will consider only very short time lags. As an example for the analysis of the synthetic aperture radar signal onboard the ENVISAT ESA satellite, the Doppler bandwidth is $\Delta f=1652 \mathrm{~Hz}$, i.e. $\Delta t=0.6 \mathrm{~ms}$. We can perform a Taylor expansion around zero at first order to describe changes of the correlation function during this small time lag:

$$
\tilde{\rho}(\boldsymbol{r}, t) \simeq \tilde{\rho}(\boldsymbol{r}, 0)+\left.t \frac{\partial \tilde{\rho}(\boldsymbol{r}, t)}{\partial t}\right|_{t=0}+\cdots
$$

with $\tilde{\rho}(\boldsymbol{r}, 0)$ the correlation function of the modified sea surface height spectrum $\tilde{S}(\boldsymbol{\xi})$ :

$$
\tilde{\rho}(\boldsymbol{r}, 0)=\tilde{\rho}_{0}(r)-\tilde{\rho}_{2}(r) \cos 2\left(\phi-\phi_{w}\right),
$$

where $\phi_{w}$ is the wind direction and $\phi$ the scattered azimuth angle. The isotropic part $\tilde{\rho}_{0}(r)$ and the anisotropic part $\tilde{\rho}_{2}(r)$ are given by:

$$
\begin{aligned}
& \tilde{\rho}_{0}(r)=\int_{0}^{\infty} \tilde{S}(\xi) J_{0}(r \xi) d \xi \\
& \tilde{\rho}_{2}(r)=\int_{0}^{\infty} \tilde{S}(\xi) \tilde{\Delta}(\xi) J_{2}(r \xi) d \xi
\end{aligned}
$$

where $J_{n}$ is the $n^{\text {th }}$ order Bessel function of the first kind and $\tilde{S}(\boldsymbol{\xi})=[1+$ $\left.\tilde{\Delta}(\xi) \cos \left(2 \phi_{w}\right)\right] \tilde{S}(\xi) / \xi$. Following the same approach, $\partial \tilde{\rho}(\boldsymbol{r}, t) /\left.\partial t\right|_{t=0}$ can be found to write as:

$$
\left.\frac{\partial \tilde{\rho}(\boldsymbol{r}, t)}{\partial t}\right|_{t=0}=\tilde{\rho}_{1}(r) \cos \left(\phi-\phi_{w}\right)-\tilde{\rho}_{3}(r) \cos 3\left(\phi-\phi_{w}\right),
$$


(a)

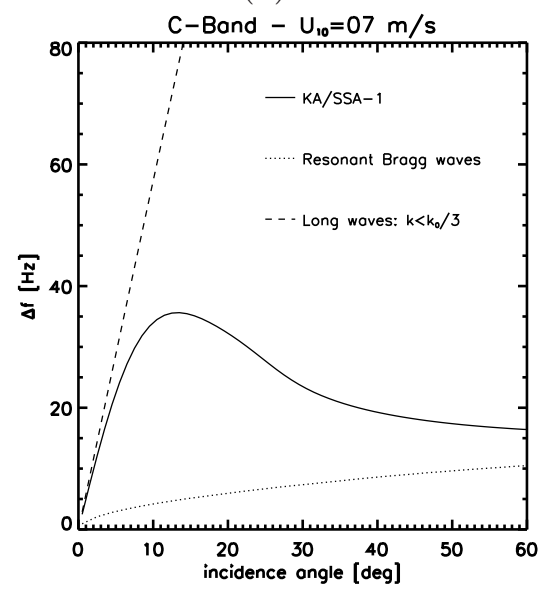

(b)

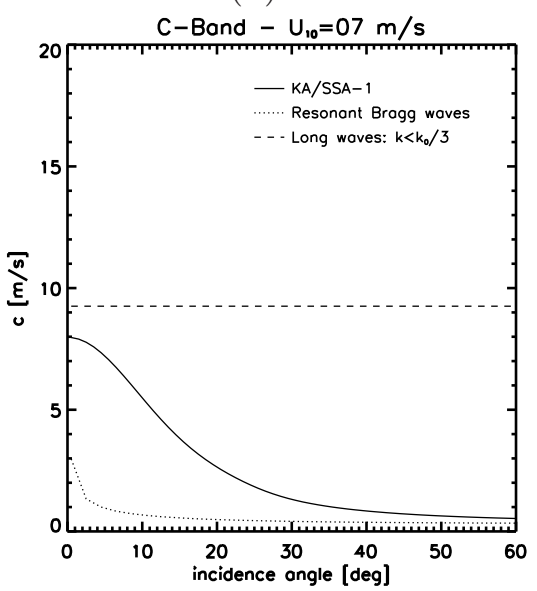

Figure 1. (a) Doppler frequency at C-band predicted using KA/SSA-1 (solid), Bragg (dotted) and geometrical (dashed) models as a function of incidence angle in up-wind configuration. Wind speed is $7 \mathrm{~m} / \mathrm{s}$, (b) Sea surface velocity projected along the line-of-sight of the radar in up-wind configuration with the same models and wind configuration than in (a).

where

$$
\begin{aligned}
& \tilde{\rho}_{1}(r)=\int_{0}^{\infty} \omega(\xi) \tilde{S}(\xi)\left[1+\frac{\tilde{\Delta}(\xi)}{2}\right] J_{1}(r \xi) d \xi \\
& \tilde{\rho}_{3}(r)=\int_{0}^{\infty} \omega(\xi) \tilde{S}(\xi) \frac{\tilde{\Delta}(\xi)}{2} J_{3}(r \xi) d \xi
\end{aligned}
$$

with $\omega(\xi)=c(\xi) \xi . c(\xi)$ is the phase speed of the surface wave at a given wavenumber $\xi \cdot \omega(\xi)$ is calculated according to the dispersion relationship:

$$
\omega(\xi)=\sqrt{g \cdot \xi+T \cdot \xi^{3}},
$$

with $g$ the acceleration of the gravity constant and $T=74.10^{-6}$ the kinematic surface tension of water. The numerical computation are done according to the numerical recipes already reported (see e.g. $[9,10]$ )

Now, the evaluation of the integral in Eq. (11) yields to a complex value. Keeping our general formulation for the expansion of the scattering matrix, Eq. (11) can be rewritten as:

$$
\sigma_{0}(\theta, \phi, t)=\left|\frac{\mathbb{N}_{0}\left(\boldsymbol{k}, \boldsymbol{k}_{0}\right)}{Q_{z}}\right|^{2} e^{i \Omega^{p q} t} e^{-Q_{z}^{2} \tilde{\rho}(0,0)} \int_{\boldsymbol{r}}\left[e^{-Q_{z}^{2} \tilde{\rho}(\boldsymbol{r}, 0)}-1\right] e^{-i \boldsymbol{Q}_{H} \cdot \boldsymbol{r}} d \boldsymbol{r}
$$


(a)

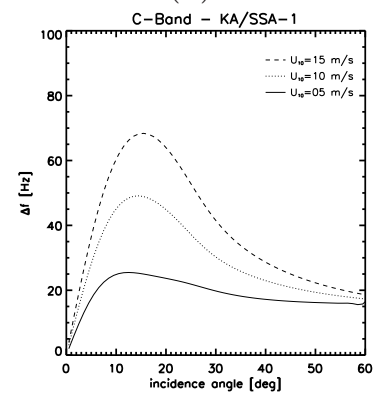

(b)

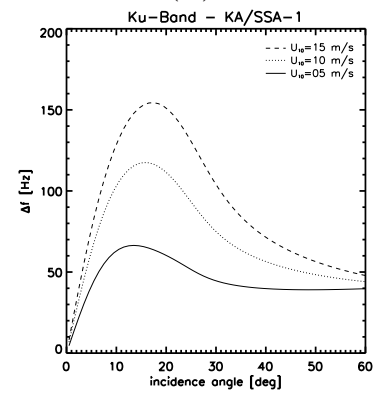

(c)

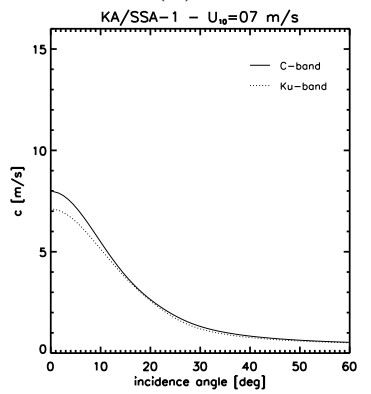

Figure 2. (a-b) Doppler frequency at (a) C- and (b) Ku-band predicted using KA/SSA-1 as a function of incidence angle in up-wind configuration. Wind speeds considered are 5 (solid), 10 (dotted) and $15 \mathrm{~m} / \mathrm{s}$ (dashed). (c) Sea surface velocity projected along the line-of-sight of the radar in up-wind configuration versus incidence angle for a $7 \mathrm{~m} / \mathrm{s}$ wind speed. C-(dotted) and $\mathrm{C}$-(solid) band are considered.

Introduction of a time dependent phase term leads to the definition of a Doppler shift induced by the distributed sea surface motions. This Doppler pulsation corresponds to an instantaneous pulsation defined as:

$$
\Omega^{p q}(\theta, \phi)=\frac{1}{t} \tan ^{-1}\left[\frac{\operatorname{Im}\left\{\sigma_{0}(\theta, \phi, t)\right\}}{\operatorname{Re}\left\{\sigma_{0}(\theta, \phi, t)\right\}}\right]
$$

Assuming a very small phase term, we can also evaluate the Doppler pulsation as:

$$
\Omega^{p q}(\theta, \phi)=\frac{1}{t}\left[\frac{\operatorname{Im}\left\{\sigma_{0}(\theta, \phi, t)\right\}}{\sigma_{0}(\theta, \phi, 0)}\right]
$$

The Doppler frequency $\Delta \mathcal{F}^{p q}$ used in the following is related to the pulsation through the relation $\Omega^{p q}=2 \pi \Delta \mathcal{F}^{p q}$.

\section{Results and Discussion}

Interestingly, according to Eq. (23), the zeroth order term of the scattering matrix expansion (i.e. $\mathbb{N}_{0}\left(\boldsymbol{k}, \boldsymbol{k}_{0}\right)$ ) will not influence the estimation of the Doppler frequency. As a direct consequence, both SSA-1 and KA would yield the same Doppler frequency. Accordingly, Doppler frequencies predicted by these models will not be polarization dependent whereas these two asymptotic theories give complete opposite results to predict the normalized radar cross-section (NRCS). Indeed, SSA-1 (KA) maximizes (minimizes) the polarization depen- 
dency for backscatter geometry. This clearly underlines the necessity of using an extended solution to better compare predictions and measurements when experimental Doppler anomalies are found polarization dependent.

Theories like SSA-2, LCA-1 or RCA enable to take into account higher orders in the scattering matrix definition and thus will lead to polarization dependent Doppler frequencies. Hereafter, we present simulations for different radar wavelengths, incidence angles, azimuth angles and wind configurations (speed and direction) using RCA [3] theory in the backscatter configuration. Indeed, this model was found to correctly reproduce the polarisation sensitivity of microwave measurements in the microwave domain [3,13]. To discuss polarization effect we also present results obtained using LCA-1 [6]. To compute the correlation function of the sea surface, we use the wind driven sea surface spectrum proposed by Elfouhaily et. al. [12]. The predicted Doppler frequencies are thus only associated with wind driven sea surface wave distributed motions.

In figure 1 (a), we present the Doppler frequency as a function of incidence angle predicted by KA/SSA-1 at C-band $\left(\lambda_{0}=5.61 \mathrm{~cm}\right)$ for a $7 \mathrm{~m} / \mathrm{s}$ wind speed. We observe that the induced Doppler shift first rapidly increases with incidence angle from zero Hertz at nadir to a maximum around $10-20^{\circ}$ (depending on the wind speed considered) and then decreases. This decrease slows down after $30^{\circ}$ to reach an asymptotic value around $60^{\circ}$. To interpret the role of the surface moving waves on the Doppler shift, we also present on the figure 1 (b) the speed of the surface elements in the line-of-sight of the radar inducing the Doppler shift. The Doppler shift $\Delta f$ and speed of surface elements in the line-of-sight of the radar $\bar{c}$ are related by the relation:

$$
\Delta \mathcal{F}=\bar{c} \frac{Q_{H}}{2 \pi},
$$

We observe the speed in the line-of-sight of the radar to decrease with increasing incidence angle. Close to nadir, the waves participating to the induced $\Delta \mathcal{F}$ correspond to larger scales which propagate faster. At higher incidence angles, the impact of waves contributing to the Doppler is dominated by shorter scales which slowly propagate. For comparisons, we also indicate on both figures the Doppler frequency associated to the speed of the resonant Bragg waves $\delta\left(Q_{H}\right)$ and the slopes of the so-called long tilting waves in the framework of the two-scale or the geometrical optics models. For this geometrical optics approximation it is often advanced that the slopes of the long waves $\left(\xi<k_{0} / 3\right)$ which contribute, and

$$
\bar{c}=\frac{\int_{0}^{k_{0} / 3} c(\boldsymbol{\xi}) \boldsymbol{\xi}^{2} S(\boldsymbol{\xi}) d \boldsymbol{\xi}}{\int_{0}^{k_{0} / 3} \boldsymbol{\xi}^{2} S(\boldsymbol{\xi}) d \boldsymbol{\xi}}
$$


(a)

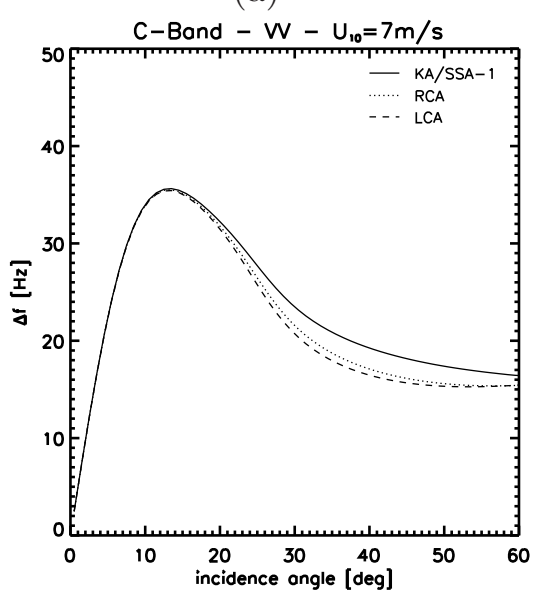

(b)

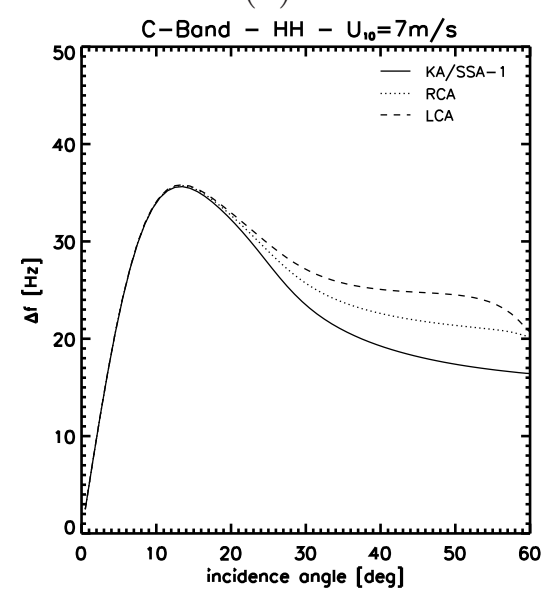

Figure 3. Doppler frequency at C-band predicted using KA/SSA-1 (solid), RCA (dotted) and LCA-1 (dashed) models as a function of incidence angle in up-wind configuration. Wind speed is 7 $\mathrm{m} / \mathrm{s}$. (a) VV polarization. (b) $\mathrm{HH}$ polarization.

The Doppler shifts induced by the resonant Bragg waves and the slopes of the longer waves indicate the asymptotic limits of the solution. We observe that indeed the result given by KA/SSA-1 model lead to predictions between these two limits. The smaller is the incidence angle, the closer to the longer wave slope solution are the simulations. As the incidence angle increases, the simulations tend toward the Bragg result. However, we observe that even at $60^{\circ}$, the model does not reach the Bragg solution. There is still an impact of non-resonant background waves in the Doppler evaluation. This is due to the multi-scale nature of the ocean surface. Interestingly, we notice that this multiscale effect is not obvious when simply comparing Bragg and SSA-1 solutions for the radar cross section evaluation at large incidence angle.

In figure 2, the Doppler frequency is evaluated for two different microwave wavelengths, (a) C- and (b) Ku-band $\left(\lambda_{0}=2.14 \mathrm{~cm}\right)$, for three wind speeds ( 5 , 10 and $15 \mathrm{~m} / \mathrm{s}$ ) in the upwind configuration as a function of incidence angle. The KA/SSA-1 solution is used for the computation here. These comparisons shows that $\Delta \mathcal{F}$ increases with the frequency. This result can appear nonintuitive. Indeed, as the frequency increases, the radar cross section is sensitive to smaller scales and thus to slower waves. Thus we could expect a smaller Doppler shift. This apparent inconsistency is only due to the relation (see Eq. (25)) between the Doppler frequency and the speed in the line-of-sight. Indeed, $Q_{H}$ has a significant impact. On figure 2 (c), we present the speed in the radar line-of-sight as a function of incidence angle in $\mathrm{C}$ - and $\mathrm{Ku}$ - band for a $7 \mathrm{~m} / \mathrm{s}$. As expected, we observe that the induced speed in the radar line-of-sight is lower 
(a)

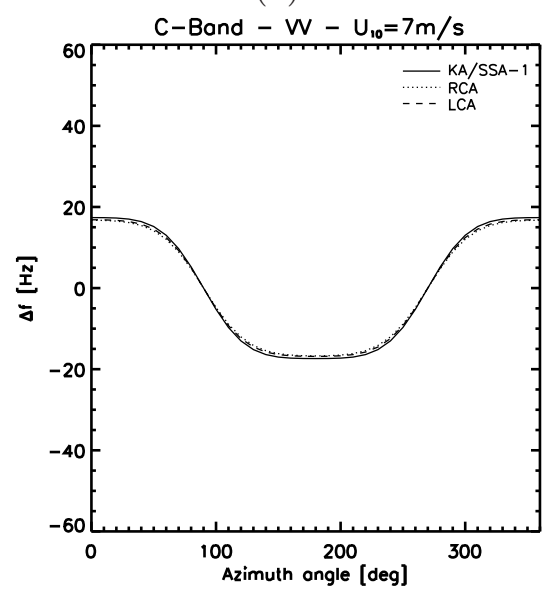

(b)

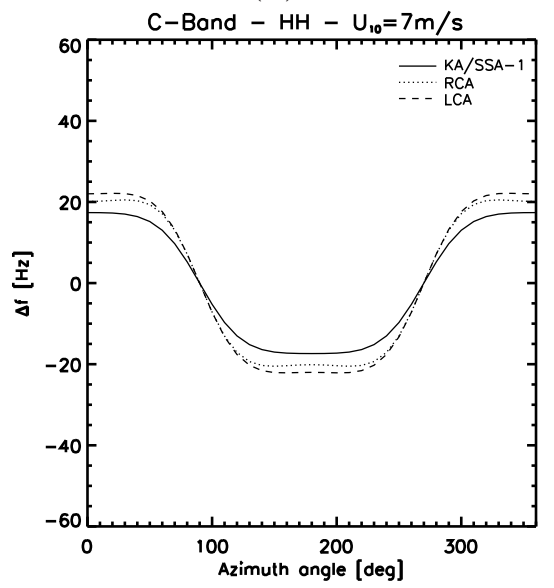

Figure 4. Doppler frequency at C-band predicted using KA/SSA-1 (solid), RCA (dotted) and LCA-1 (dashed) models as a function of azimuth angle at $50^{\circ}$. Wind speed is $7 \mathrm{~m} / \mathrm{s}$. (a) VV polarization. (b) $\mathrm{HH}$ polarization.

in $\mathrm{Ku}$ - than in C-band. These simulations also indicates that the Doppler shift increases with wind speed. Compared to near-nadir configuration, a saturation effect is obtained for large incidence angles $\left(>30^{\circ}\right)$. At these larger incidences, the radar is more sensitive to the shorter gravity and capillary waves which are rapidly saturated with increasing wind speed. As their relative weight to the Doppler signal is more important than larger and faster waves at these large incidences, the weak wind speed dependency is expected.

In figure 3, we present the behavior of the Doppler shift as a function of incidence angle using LCA-1 and RCA models for a $7 \mathrm{~m} / \mathrm{s}$ wind speed to take into account polarization effects through the first order term effect in the scattering expansion. Figure 3 (a) corresponds to VV-polarization and figure 3 (b) to HH. KA/SSA-1 results are also presented for comparisons. As anticipated from the theory, we observe a polarization dependency. The predicted wind induced Doppler frequency is always found larger in $\mathrm{HH}$ than in VV polarization. This result is expected as the radar signal is more sensitive to the smaller waves in VV polarization than in HH. This comes from the kernel filter effect on the surface elevation. As, shorter gravity ocean waves are slower, the natural consequence is that the relative weight of the larger faster propagating waves in the calculation of $\Delta \mathcal{F}^{p q}$ is more significant in $\mathrm{HH}$ than in VV polarization. As the phase speed of the waves increases with increasing surface wavelength, $\Delta \mathcal{F}^{H H}>\Delta \mathcal{F}^{V V}$. Also, $\Delta \mathcal{F}^{V V}$ and $\Delta \mathcal{F}^{H H}$ predicted by RCA/LCA are always respectively lower and higher than the predicted Doppler frequency given by the zeroth order solution for the scattering matrix expansion. Focusing on RCA 
and LCA results, we observe that $\Delta \mathcal{F}_{\mathrm{LCA}}^{H H}>\Delta \mathcal{F}_{\mathrm{RCA}}^{H H}$ and $\Delta \mathcal{F}_{\mathrm{LCA}}^{V V}<\Delta \mathcal{F}_{\mathrm{RCA}}^{V V}$. This can be explained by the construction of the two models. The first order term of the scattering matrix expansions is respectively defined for LCA-1 as:

$$
N_{1}\left(\boldsymbol{k}, \boldsymbol{k}_{0} ; \boldsymbol{\xi}\right)=-i\left[\mathbb{B}\left(\boldsymbol{k}, \boldsymbol{k}_{0} ; \boldsymbol{\xi}\right)-\mathbb{K}\left(\boldsymbol{k}, \boldsymbol{k}_{0}\right)\right],
$$

and for RCA:

$$
N_{1}\left(\boldsymbol{k}, \boldsymbol{k}_{0} ; \boldsymbol{\xi}\right)=-i\left[\mathbb{B}\left(\boldsymbol{k}, \boldsymbol{k}_{0} ; \boldsymbol{\xi}\right)-\mathbb{K}\left(\boldsymbol{k}, \boldsymbol{k}_{0}\right)\right] \delta\left(\boldsymbol{\xi}=\boldsymbol{Q}_{H}\right),
$$

where $\delta$ is the dirac function. As a remarkable property, $\mathbb{B}\left(\boldsymbol{k}, \boldsymbol{k}_{0} ; \boldsymbol{\xi}\right)-$ $\mathbb{K}\left(\boldsymbol{k}, \boldsymbol{k}_{0}\right) \propto \boldsymbol{\xi}^{2}$, and in the framework of LCA theory, the correction is associated to the surface curvature. For an ocean surface, the relative weight of the smaller and slower waves is larger (respectively lower) in VV (respectively $\mathrm{HH}$ ) than with RCA. This explains the difference between the two predicted Doppler frequency anomalies.

In figure 4, we present the Doppler shift as a function of the relative azimuth angle between the radar look direction and the wind speed in $\mathrm{VV}$ (a) and $\mathrm{HH}$ (b) polarization given by KA/SSA-1, LCA and RCA for a $50^{\circ}$ incidence angle. As the standard convention, $0^{\circ}, 180^{\circ}, 90^{\circ}$ and $270^{\circ}$ stand for up-, downand cross-wind directions, respectively. As anticipated, we observe that when the wind direction is perpendicular to the antenna direction and the radial component of the wind is zero, the Doppler shift is zero. Also, when the mean motion is directed toward the radar antenna (up-wind case) the Doppler frequency is positive whereas it changes sign under down-wind conditions. The absence of difference between the up- and down-wind configuration is expected as the Gaussian hypothesis for the surface height statistics was adopted for the NRCS derivation.

As a first exercice of validation, we use two data sets of Doppler anomalies derived from the ASAR instrument onboard the ENVISAT satellite. The first data set was obtained during one month of measurements. It represents more than 30000 values of Doppler anomalies. It was already used in [1]. The details about the SAR images processing and the co-located wind speed analysis can be found in $[1,2]$. The radar frequency is $5.35 \mathrm{GHz}$, the incidence angle $23^{\circ}$ and the polarization VV. The second data set was processed with the same methodology and correspond to measurements at $33.5^{\circ}$ in $\mathrm{VV}$ and $\mathrm{HH}$ polarizations. In both cases, the Doppler shift is evaluated over a $10 \times 10 \mathrm{~km}$ area. Such a resolution cell and global measurements for the Doppler shift estimation is expected to minimize the current effects. It is thus adapted to investigate the wind effect on the Doppler shift. A co-localisation with predicted wind speed and direction then helps to investigate the relationship between the radial wind speed component and the Doppler shift. In figure 5, we present the 
(a)

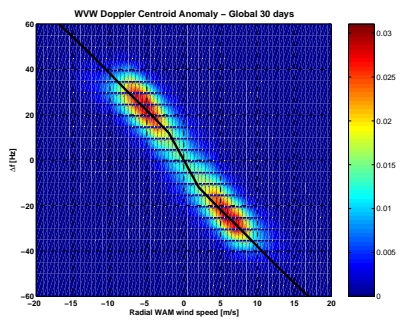

(b)

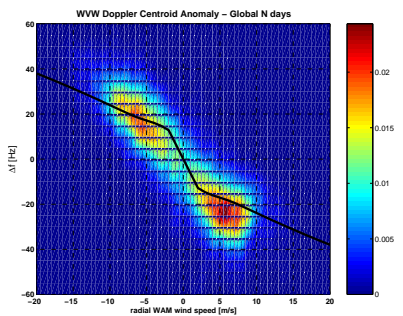

(c)

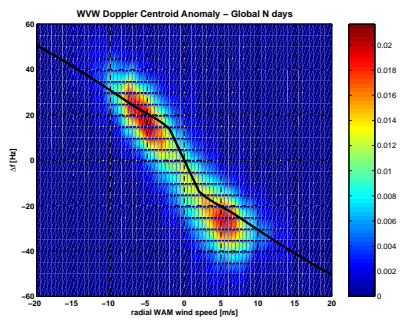

Figure 5. Analysis of Doppler on a global scale. Joint distributions of observed Doppler anomaly and line-of-sight analyzed winds from numerical model (color) and predictions given by RCA model for up-wind (positive value) and down-wind values (negative values). (a) VV polarization and $23^{\circ}$ as incidence angle, (b) $\mathrm{VV}$ polarization and $33.5^{\circ}$ as incidence angle, (c) $\mathrm{HH}$ polarization and $23^{\circ}$ as incidence angle

result given by RCA considering only a wind speed configuration in the upand down-wind direction. The wave spectrum used as input is again the one proposed by [12]. Given this sea surface description, the overall comparison with the data is rather good. Discrepancies must certainly be attributed to the sea surface description at low winds. As measured and predicted, the Doppler frequencies are smaller at $33.5^{\circ}$ incidence angle than at $23^{\circ}$, in $\mathrm{VV}$ polarization. It is also found a slight polarization dependency at $33.5^{\circ}$ incidence angle. For the most populated samples, i.e. $7 \mathrm{~m} / \mathrm{s}$, the RCA predictions are in excellent agreement. Such a result is consistent with NRCS analysis [13], especially in terms of predicted NRCS polarization ratio measurements.

\section{Summary and Conclusion}

In this paper, developments are conducted to use and apply the latest improvements suggested in the field of the asymptotic electromagnetic modeling. Under Gaussian statistics, time dependent asymptotic solutions have been derived to define a Doppler frequency and the associated measured motion.

As demonstrated, these developments help to better understand time dependent radar backscattered signal sensitivities to both sea surface characteristics and instrumental configurations. As obtained, Doppler shifts will be wavelength and incidence angle dependent. Using extended scattering solution polarization dependency is also predicted.

At low incidence angle, the sea surface induced Doppler shifts are largely dominated by the mean velocity of the larger filtered sea surface slope components. At larger incidence angles, predicted Doppler shifts will be more dominated by shorter, and thus slower, surface roughness elements. However, at larger incidence angles, predictions do not necessarily converge toward reso- 
nant Bragg solutions. These results confirm the importance of the multi-scale nature of the ocean surface. This result is consistent with previous NRCS analysis.

As compared with measurements, the weak but systematic polarization sensitivity of Doppler shifts can only result from the use of extended asymptotic scattering solutions. In particular, both KA and SSA-1 solutions would fail to reproduce these measured sensitivity. As presented here, the local and resonant curvature solution, through a phase perturbation formalism, yield polarization sensitivity. SSA-2 solution would have also produced this desired polarization sensitivity.

As foreseen, the use of combined Doppler and radar cross-section measurements can certainly help the development of a consistent inversion of instantaneous sea surface geometry and kinematic properties. As an optimal configuration, to identify and minimize the wind induced surface motion, the use of dual-polarized instrument is recommended. Indeed, according to the different asymptotic electromagnetic models, the polarization sensitivity can certainly be attributed to the shorter scale roughness elements. As these elements are the most sensitive to wind changes, the use of dual-polarized measurements will help to instantaneously evaluate and map both the strength and mean motion of these elements over the illuminated ocean scenes.

\section{References}

[1] Chapron, B., Collard, F. and Ardhuin, F., 2005, Direct measurements of Ocean Surface Velocity from Space: Interpretation and Validation, Journal of Geophysical Research, 110(C07008), doi:10.1029/2004JC002809.

[2] Chapron, B., Collard, F. and Kerbaol, V., 2004, Satellite Synthetic Aperture Radar Sea Surface Doppler Measurements, in Proceeding of the 2nd Workshop on Coastal and Marine Applications of $S A R$, ESA SP-565, 133-140,

[3] Mouche, A.A., Chapron, B., and Reul, N., 2007, A Simplified Asymptotic Theory for Ocean Surface Electromagnetic Waves Scattering, Accepted for publication in Waves in Random and Complex Media.

[4] Elfouhaily, T. and Guerin, C.-A., 2004, A critical survey of approximate wave theories from random rough surfaces. Waves in Random Media, 14, R1-R40.

[5] Irisov, V.G., 1994, Small-slope expansion for electromagnetic-wave diffraction on a rough surface. Waves in Random Media, 4, 441-452.

[6] Elfouhaily, T., Guignard, S., Awadallah, R. and Thompson, D. R., 2003, Local and non-local curvature approximation: A new asymptotic theory for wave scattering. Waves in Random Media, 13(4), 321-337.

[7] Berman, D.H. and Dacol, D.K., 1990, Manifestly reciprocal scattering amplitudes for rough surfaces interface scattering, Journal of the Acoustical Society of America, 87(5), 2024-2032.

[8] Voronovich, A.G. and Zavorotny, V.U., 2001, Theoritical model for scattering of radar signals in $\mathrm{Ku}$ and $\mathrm{C}$ bands from a rough sea surface with breaking waves. Waves in Random Media, 11, $247-269$

[9] Bourlier, C., 2005, Azimuthal harmonic coefficients of the microwave backscattering from a nonGaussian ocean surface with the first-order SSA model. IEEE Transaction on Geoscience and Remote Sensing, 42(11), doi:10.1109/TGRS.2004.836874.

[10] Reul, N., Tenerelli, J., Chapron, B. and Waldteufel, P., 2006. Modeling Sun glitter at L-band for the sea surface salinity remote sensing with SMOS. accepted for publication in IEEE Transaction on Geoscience and Remote Sensing, MicroRad06 Special issue paper 
[11] Voronovich, A.G., 1994, Small Slope Approximation for electromagnetic wave at a rough interface of two dielectric half-spaces, Waves in Random Media,, 4, 337-367.

[12] Elfouhaily, T., Chapron, B., Katsaros, K. and Vandemark, D., 1997, A unified directional spectrum for long and short wind driven waves, Journal of Geophysical Research, 102(C7), 1578115796, doi:10.1029/97JC00467.

[13] Mouche, A.A., Chapron, B., Reul, N., Hauser, D. and Quilfen Y., 2007, Importance of the sea surface curvature to interpret the normalized radar cross-section, Submitted in Journal of Geophysical Research. 